\title{
GCU
}

Glasgow Caledonian

University

University for the Common Good

\section{A repository of resources and exemplars for the cloud curriculum}

Paterson, James; Adams, Joshua; Hainey, Brian; Nazir, Sajid

Published in:

Computing Education Practice 2021 (CEP '21)

DOI:

10.1145/3437914.3437971

Publication date:

2021

Document Version

Author accepted manuscript

Link to publication in ResearchOnline

Citation for published version (Harvard):

Paterson, J, Adams, J, Hainey, B \& Nazir, S 2021, A repository of resources and exemplars for the cloud curriculum. in Computing Education Practice 2021 (CEP '21). Association for Computing Machinery (ACM), pp. 9-12, 5th Computing Education Practice 2021, Durham, United Kingdom, 7/01/21. https://doi.org/10.1145/3437914.3437971

\section{General rights}

Copyright and moral rights for the publications made accessible in the public portal are retained by the authors and/or other copyright owners and it is a condition of accessing publications that users recognise and abide by the legal requirements associated with these rights.

Take down policy

If you believe that this document breaches copyright please view our takedown policy at https://edshare.gcu.ac.uk/id/eprint/5179 for details of how to contact us. 


\section{A Repository of Resources and Exemplars for the Cloud Curriculum}

\author{
James Paterson \\ Department of Computing \\ Glasgow Caledonian University \\ Glasgow, Scotland \\ UK \\ James.Paterson@gcu.ac.uk \\ Brian Hainey \\ Department of Computing \\ Glasgow Caledonian University \\ Glasgow, Scotland \\ UK \\ b.hainey@gcu.ac.uk
}

\author{
Joshua Adams \\ Donald R. Tapia College of Business \\ CS Department \\ Saint Leo University \\ Saint Leo, Florida \\ USA \\ joshua.adams03@saintleo.edu \\ Sajid Nazir \\ Department of Computing \\ Glasgow Caledonian University \\ Glasgow, Scotland \\ UK \\ sajid.nazir@gcu.ac.uk
}

\begin{abstract}
The increasing adoption of cloud computing is driving a demand for skills which needs to be addressed in higher education. However, the nature of the field presents significant challenges for educators. The body of knowledge associated with cloud computing is not well established, and many educators may not be comfortable with cloud computing concepts. Furthermore the services offered by the major cloud service providers (CSPs) tend to evolve rapidly.On the other hand, there is a wealth of up-to-date teaching and learning material available, much of it supported by the CSPs and provided free to students. The aim of the resource described here is to help instructors to define learning goals and identify suitable materials when designing their own cloud curriculum. Through the efforts of a series of ITiCSE Working Groups, a set of Knowledge Areas (KAs) for Cloud has been defined, and a canonical list of Learning Objectives (LOs) which align with the KAs has been developed. A repository has been created which contains: the definitive set of KAs and LOs; information on available teaching resources that align with the LOs; and exemplar modules that demonstrate the viability of using these to construct units of learning. The ongoing development of the repository and
\end{abstract}

Permission to make digital or hard copies of all or part of this work for personal or classroom use is granted without fee provided that copies are not made or distributed for profit or commercial advantage and that copies bear this notice and the full citation on the first page. Copyrights for components of this work owned by others than ACM must be honored. Abstracting with credit is permitted. To copy otherwise, or republish, to post on servers or to redistribute to lists, requires prior specific permission and/or a fee. Request permissions from permissions@acm.org.

CEP '21, January 7, 2021, Durham, United Kingdom

(c) 2021 Association for Computing Machinery.

ACM ISBN 978-1-4503-8959-4/21/01 .. \$15.00

https://doi.org/10.1145/3437914.3437971 the use of the resources is described. It is proposed that the repository will form the core of and be maintained by an online community.

CCS Concepts: • Applied computing $\rightarrow$ Education; $•$ Computer systems organization $\rightarrow$ Cloud computing.

Keywords: Cloud Computing, Education, Computer Science, Curriculum Development, Distributed Computing.

\section{ACM Reference Format:}

James Paterson, Joshua Adams, Brian Hainey, and Sajid Nazir. 2021. A Repository of Resources and Exemplars for the Cloud Curriculum. In Computing Education Practice 2021 (CEP '21), fanuary 7, 2021, Durham, United Kingdom. ACM, New York, NY, USA, 4 pages. https: //doi.org/10.1145/3437914.3437971

\section{Introduction}

The adoption of cloud computing in industry is accelerating rapidly and consequently there is a growing demand for higher education graduates to have an understanding of the significance of cloud technology and practical skills in the use of the cloud as a key platform for providing solutions to business problems. According to LinkedIn data cloud computing was the top "hard skill" required by companies in 2019 [9].

Since the field has evolved at such a rapid rate, the body of knowledge in cloud computing is not well established or defined at a level of detail that is useful for instructors who need or want to incorporate the cloud in their curriculum. General computing curriculum guidelines, such as the ACM Computer Science 2013 guidelines [8] do not typically provide such a granular view of cloud computing.

Hands-on experience is dependent on access to the environments provided by the CSPs, which are often subject to rapid change. However, there is a great deal of learning 
material available which is actively kept up to date, in many cases by the CSPs themselves, with much of this freely available to students. Therefore, a strong case exists for the use of vendor learning material to support practical teaching of cloud computing rather than using textbooks or instructors maintaining their own materials.

A series of three ITiCSE Working Groups [1, 5, 6] has begun addressing challenges involved in incorporating cloud computing in higher education by initially defining a body of knowledge for cloud computing in terms of a comprehensive set of Knowledge Areas (KAs) and Learning Objectives (LOs), surveying publicly available learning materials and creating a mechanism for instructors to share learning materials. The most recent Working Group mapped publicly available learning materials to LOs and produced module exemplars to demonstrate how this knowledge base can be used to design curriculum specific modules for inclusion in computing courses. This work has culminated in the creation of a repository that includes the body of knowledge, information on accessing mapped learning content and a set of exemplar modules. This paper describes how instructors can make use of this repository in to support their own curriculum and proposes how the community can contribute to maintaining the repository as valuable resource.

\section{Related Work}

Sets of key topics or knowledge areas have been defined previously for cloud computing. Brievold and Crnkovic [3] identify a set of five key knowledge areas in cloud computing:i) cloud fundamentals, ii) architecting for the cloud, iii) design for resilience, iv) cloud security and compliance, and v) migrating to the cloud. They note the need to teach enduring principles within a rapidly evolving area in which products and vendors can be short-lived. Sommerville [10] defines a similar set of key topics with a focus on software engineering. Guo and Koufakou [7] define a body of knowledge for a course guided by the requirements of an industryrecognised Amazon Web Services (AWS) certification [2]. Demchenko et al. [4] report development and teaching of courses in cloud based data analytics based on a comprehensive framework which provides support for teaching in a specific field, data science in this case, that includes including learning outcomes, a body of knowledge and a model curriculum.

\section{The Repository}

The 2019 Working Group initially of created a repository to house a collection of materials that could be used in teaching cloud computing, and to allow new materials to be shared [6]. GitHub was chosen as a platform for this as it provides a standard infrastructure for storing structured information and a collaborative platform with well-known processes for uploading and updating content. The Cloud-ComputingCurriculum repository ${ }^{1}$ is an evolution of the original repository, restructured to provide a resource to support curriculum design, in addition to the original role of hosting and sharing learning materials.

The repository is a work in progress, but the main content areas are in place and their structure defined, and methods for contributing to the repository have been determined. The top-level content areas are described in the following sections.

\subsection{Core: Knowledge Areas and Learning Objectives}

The Core content area contains the body of knowledge as developed by the Working Groups. This is presented in two levels - KAs and LOs. The top level KAs are listed in table 1.

Table 1. The cloud computing Knowledge Areas

\section{Knowledge Area Definition}

Fundamental Cloud Introduces the fundamental conConcepts (FCC) cepts and gives a general overview of the cloud.

Computing Abstrac- Comprises different encapsulation tions on the Cloud mechanisms to abstract computing (CAC)

Storage Resources on Discusses, compares and utilizes the Cloud (SRC) the various cloud storage methods/types as well as the suitability of the storage types for a specific scenario.

Networking Re- Covers general cloud-related consources on the Cloud cepts for networking.

(NRC)

Cloud Elasticity and Discusses vertical and horizontal Scalability (CES) scaling configurations, with associated cost/performance trade-offs for various cloud infrastructure methods (IaaS, PaaS, SaaS).

Fault Tolerance, Re- Addresses the properties of a cloudsilience and Reliabil- based solution to operate in a stable ity (FTRR)

Cloud, Monitoring and Maintenance (CMM)

Cloud Orchestration (CO) and consistent way, according to the service expectations of the cloudbased solution.

Focuses on how the cloud changes the way software applications are developed, run and monitored and managed.

Covers how the cloud impacts deployment methods and frameworks for how software applications are developed and deployed.

$\overline{{ }^{1} \text { https://github.com/cloudedrepo/Cloud-Computing-Curricula }}$ 
Software Development using Cloud APIs (SDCA)

Cloud Programming Models and Frameworks (CPMF) Service Oriented Architecture (SOA)

Cloud Security, Privacy, Policy and Ethics (CSPPE)

IoT, Mobile, Edge and the Cloud (IoTMEC)

Cloud-based Artificial Intelligence and Machine Learning (CAIML)

Each KA has a set of LOs associated with it. These LOs evolved from a first iteration, which was defined along with the KAs by the 2018 Working Group [5]. The original LOs were based primarily on analysis of a survey of cloud courses at various types of higher education institutions in different geographic regions. The 2020 Working Group mapped these LOs to those of a range of current learning materials, and in the process discovered additional LOs [1]. To be included in the list, LOs had to: map to KAs and specific cloud concepts; include foundational knowledge; and have wide applicability and not just be a tool of a single CSP.

Note that while the repository will contain information about provider-specific learning resources, the body of knowledge itself is provider-neutral. It is anticipated that for a given LO a choice of materials may be available to teach that LO using a range of cloud platforms. LOs are classified as either "conceptual" or "experiential" according to their emphasis on knowledge or on hands-on skills. The repository entry for each LO will contain links to information elsewhere in the repository on material that can be used to teach that LO. Each LO is assigned a short code to identify it, the KA it relates to and whether it is conceptual or experiential. The full list will be available in the final report from the 2020 Working Group.

\subsection{Materials}

The Materials content area contains a potentially large set of entries which describe available learning materials. For each resource in the set, the information stored will include the following:

- type of resource - for example lecture slides, lab assignments summary

- access - for example, some material is provided free by CSPs but access may depend on education status and must be requested

- intended level

- cloud vendor - where the material is provider-specific

- prerequisites

- tags/keywords

\section{Contributing}

We have made available a simplified process for material contribution taking into account the skills of contributors. Contributors familiar with GitHub, can follow through the process of creating a new page, updating links to new material, and issuing a pull request. As this process may not be convenient for those not familiar with GitHub, any contributor can share content by filling relevant details on a Google Form which will be made available. In either case, the provided information will be reviewed by the administrators for inclusion in the repository.

\section{Using the Repository}

Instructors coming to this repository may wish to simply browse for content, in which case they can browse by LOs, browse by learning materials, or simply search the content. However, we envisage a more structured scenario whereby an instructor has in mind an intended purpose and scope for a module or course that they wish to design.

This would typically involve deciding on, as a minimum, a set of LOs and a set of module topics to be covered that would lead to the achievement of those LOs. The instructor may start with the top level KAs and identify which of those are relevant to the module. Then, they can browse the LOs for each of those KAs and select a set of LOs which match the overall aim of the module and provide a scope suitable for the level and size of the module. The classification of conceptual and experiential LOs will aid the selection of a suitably balanced set of LOs.

Once the LOs have been selected, the instructor can then view a range of learning materials which have been identified as relevant to those LOs. While the LOs are not providerspecific, in many cases a module will be taught mainly using a specific cloud provider's platform, using learning materials specific to that provider. By reviewing the available materials 
the instructor can decide on the platform(s) to be used in teaching the module, identify suitable learning material and define a list of module topics which can be delivered using that material.

\section{Exemplar Modules}

A set of exemplar modules has been created by Working Group members and these are included in the repository to provide further guidance on the intended usage of the resource. The development of these exemplars followed the process described in section 5 . This also provided a means to evaluate the usefulness of the repository. It was found that it was possible to start with a range of intents, from broad coverage of the 'Fundamentals of cloud Computing' at an introductory level to a more specialised 'Introduction to Big Data Analytics using Cloud Services' module, and to define a suitable set of LOs. It was then found to be possible to construct a viable set of module topics for each based on the LOs and the learning materials linked to them. It was anticipated that multiple different sets of module topics can be defined for a single set of LOs. This was demonstrated in the creation of the 'Fundamentals of Cloud Computing' exemplar in which the available learning material was found to guide two distinct topic lists, one focusing on a specific platform, the other largely platform agnostic. It was also noted that $\mathrm{LO}$ short codes that distinguish conceptual/experiential were useful in selecting a balanced set for a module.

\section{Community Building}

We envision an organic growth of the repository through open contributions and administration by a core team. The value of the repository as a resource for educators will depend on continuing maintenance of its content, through the growth of a community with a shared interest in meeting the skills needs related to cloud computing. Contributors may include academics and industry representatives. In particular, the Working Groups included members from two of the main CSPs, so there is already industry interest in the project. The core team will be responsible for reviewing contributions for inclusion, and will also review the KAs and LOs going forward, in consultation with the community, to ensure the currency of the body of knowledge.

\section{Conclusions}

Cloud computing is a rapidly evolving field and despite the availability of a wealth of learning and teaching material, mainly from the major cloud service providers, developing a well balanced syllabus can be a daunting task. This paper describes a repository of resources and exemplar modules to address the challenge of creating a cloud curriculum based on a knowledge area. The repository is a result of three years of efforts of an ITiCSE Working Group to identify the knowledge areas, decide on the learning objectives, and to map learning materials to these. The repository provides a useful organised resource on cloud computing learning material for academics to search and choose the material relevant to their modules. It is expected that the repository will grow over time with useful contributions of the cloud computing community to reflect the current state of the technologies.

\section{References}

[1] Joshua Adams, Brian Hainey, Laurie White, Derek Foster, Narine Hall, Mark Hills, Sara Hooshangi, Karthik Kuber, Sajid Nazir, Majd Sakr, Lee Stott, and Carmen Taglienti. 2020. Cloud Computing Curriculum: Developing Exemplar Modules for General Course Inclusion. In Proceedings of the 2020 ACM Conference on Innovation and Technology in Computer Science Education (Trondheim, Norway) (ITiCSE '20). Association for Computing Machinery, New York, NY, USA, 510-511. https://doi.org/10.1145/3341525.3394992

[2] Amazon Web Services, Inc. 2020. Prepare for your AWS Certification Exams. https://aws.amazon.com/certification/certification-prep/. https://aws.amazon.com/certification/certification-prep/ Retrieved 19 June 2020.

[3] Hongyu Pei Breivold and Ivica Crnkovic. 2014. Cloud Computing education strategies. In 2014 IEEE 27th Conference on Software Engineering Education and Training (CSEE\&T). IEEE, Klagenfurt, Austria, 29-38.

[4] Yuri Demchenko, Zhiming Zhao, Jayachander Surbiryala, Spiros Koulouzis, Zeshun Shi, Xiaofeng Liao, and Jelena Gordiyenko. 2019. Teaching DevOps and Cloud Based Software Engineering in University Curricula. In 2019 15th International Conference on eScience (eScience). IEEE, 548-552.

[5] Derek Foster, Laurie White, Joshua Adams, D. Cenk Erdil, Harvey Hyman, Stan Kurkovsky, Majd Sakr, and Lee Stott. 2018. Cloud Computing: Developing Contemporary Computer Science Curriculum for a Cloud-First Future. In Proceedings Companion of the 23rd Annual ACM Conference on Innovation and Technology in Computer Science Education (Larnaca, Cyprus) (ITiCSE 2018 Companion). Association for Computing Machinery, New York, NY, USA, 130-147. https://doi.org/10.1145/3293881.3295781

[6] Derek Foster, Laurie White, D. Cenk Erdil, Joshua Adams, Amadeo Argüelles, Brian Hainey, Harvey Hyman, Gareth Lewis, Sajid Nazir, Van Nguyen, Majd Sakr, and Lee Stott. 2019. Toward a Cloud Computing Learning Community. In Proceedings of the Working Group Reports on Innovation and Technology in Computer Science Education (Aberdeen, Scotland Uk) (ITiCSE-WGR '19). Association for Computing Machinery, New York, NY, USA, 143-155. https://doi.org/10.1145/3344429.3372506

[7] Dahai Guo and Anna Koufakou. 2018. A comprehensive and hands-on undergraduate course on cloud computing. In Proceedings of the ASEE Southeastern Section Conference.

[8] ACM Inc. [n.d.]. ACM Curricula Recommendations. https://www.acm. org/education/curricula-recommendations. (Accessed on 10/16/2020).

[9] Paul Petrone. 2019. The Skills Companies Need Most in 2019 - And How to Learn Them. https://www.linkedin.com/business/learning/ blog/top-skills-and-courses/the-skills-companies-need-most-in2019-and-how-to-learn-them. Retrieved 15 October 2020.

[10] Ian Sommerville. 2013. Teaching cloud computing: a software engineering perspective. Journal of Systems and Software 86, 9 (2013), $2330-2332$. 